\title{
Analysis of the Intensity of Trading on the Ukrainian Stock Market
}

\author{
Maryna Iurchenko \\ Department of Information Systems in \\ Economics \\ Chernihiv National University of \\ Technology \\ Chernihiv, Ukraine \\ http://orcid.org/0000-0001-8992-6093
}

\author{
Lyudmyla Remnova \\ Department of Finance, Banking and \\ Insurance \\ Chernihiv National University of \\ Technology \\ Chernihiv, Ukraine \\ http://orcid.org/0000-0002-5973-591
}

\author{
Olena Gonta \\ Department of Finance, Banking and \\ Insurance \\ Chernihiv National University of \\ Technology \\ Chernihiv, Ukraine \\ http://orcid.org/0000-0001-6434-357X
}

\begin{abstract}
In this research, we present a new approach for analysis of the intensity of trading on the Ukrainian stock market. In order to model the operations throughout the day, the non-homogeneous Poisson process with some unknown intensity function is used. The proposed approach is tested on the high frequency data concerning the trading operations associated with UX-index assets, with the unknown rate function estimated by kernel-based method with Epanechnikov kernel. From the analysis, it can be clearly seen that "the lunch time effect" is noticeable in the intensity profile, as well as the "day-of-the-week" effect, i.e. trading activity on the market depends both on the time of the day and day of the week with high statistical significance. This yields that stock price models with day-based switching correspond to reality better in comparison to standard models.
\end{abstract}

Keywords—stock market, non-homogeneous Poisson process, trading, kernel estimation

\section{INTRODUCTION}

In conditions of modern economy, the stock market plays a key role in the mechanism of attracting investments both to the private business and to the state budget. This fact, as well as Ukraine's integration into the world economic space, the emergence of exchange trade institutions in the Ukrainian realities and the impact of financial and economic players of the world level, have resulted in a significant increase in interest in new types of risky assets and, consequently, in demand for new mathematically valid methods that allow to maximize returns of investments in the latter. However, it should be noted that the volume of risky financial instruments trading on the stock market of Ukraine is still extremely small in comparison with the demand for state risk-free securities (see Fig.1). This leads to relatively low efficiency of the domestic stock market as a source of investment for private business.

The purpose of this study is to develop a method for analyzing the intensity of trades on the stock market. Using it, we also investigate the daily flow of transactions on the Ukrainian Stock Exchange (shares that form the UX stock index are considered, see [1] for more details), as well as to analyze the indicators related to the number of transactions for the securities mentioned above.

Another important goal of this research is to build a continuous time model for the dynamics of transaction occurrence (rather than price dynamics) on the stock market that captures the well-known "day-of-the-week" effect as well as changes in investors' activity throughout the day. Such model could be used as a basis for more sophisticated multi-level approaches (e.g., it is evident that price volatility depends on the intensity of trades, so the stochastic volatility price models with the volatility random process depending on the trade intensity reflects the reality better in comparison to standard approaches).

Volume of trading for different types of assets, $\%$

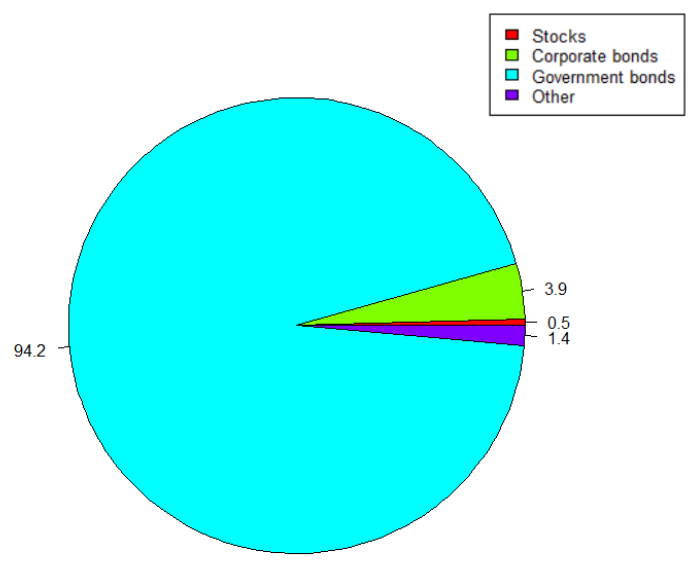

Fig. 1. Ratio of trading volumes (in UAH) for various types of assets on Ukrainian stock market. The pie chart is based on data from [2]

The "day-of-the-week" effect is widely known and extensively studied for foreign financial markets. Wellknown classical research [3], conducted in 1980, reported that the mean returns for the S\&P500 index were higher on Friday in comparison to other days. To illustrate the range of existing studies on that topic (without trying to list all possible references), we recall [4-8] and references therein.

Note that in all of the works mentioned above, authors used various time series techniques (such as ARCH, GARCH or T-ARCH models) in order to analyze the returns of the assets with the stock price as the main data source, while our approach concerns the transaction occurrence without any direct reference to price fluctuations. In such framework, it is relevant to use the non-homogeneous Poisson model and non-parametric estimation. Such 
approach is new and allows to take into account the changes in trading intensity throughout the day, i.e. the characteristics that is poorly reflected in standard linear time series methods.

\section{NON-HOMOGENEOUS POISSON MODEL FOR INTENSITY OF TRANSACTIONS}

The natural model for transaction volume dynamics on the stock market is the compound (or mixed) process [9]:

$$
X_{t}=\sum_{i=1}^{N_{t}} \xi_{i}, \quad t \in[0, T]
$$

where $N=\left\{N_{t}, t \in[0, T]\right\}$ is the counting process with $N_{t}$ interpreted as the total number of transactions up to time $t$, $\xi_{i}$ are i.i.d. random variables representing the volume (i.e. the quantity of traded stocks in pieces) of the $i$-th transaction, and $T$ is a time horizon.

\section{A. Non-homogeneous compound Poisson process}

A common choice for the counting process $N$ is the Poisson process with some intensity $m$ (with $m$ being a $\sigma$ finite measure on $[0, T]$ ), i.e. the stochastic process that satisfies the following three assumptions:

- $\quad N_{0}=0$;

- $\quad N$ is the process with independent increments;

- the increments $N_{t}-N_{s}, 0 \leq s<t \leq T$, have a Poisson distribution with parameter $m((s, t])$, i.e.

$$
\mathrm{P}\left(N_{t}-N_{s}=k\right)=\frac{e^{-m((s, t])}(m((s, t]))^{k}}{k !},
$$

with $k$ being a nonnegative integer. Such choice of the counting process can be explained by the fact that the trajectories of the Poisson process are right continuous for $t \geq 0$, have limits from the left for $t>0$ and are constant between the unit jumps, which is natural for the counting process.

It should be noted that if $m$ is the Lebesgue measure scaled with some parameter $\lambda$, i.e. $m((s, t])=\lambda(t-s)$, the Poisson process is called homogeneous (for more detail on Poisson models and their application in finance and insurance, see, for example, classical books [10] and [11]). However, despite being mathematically convenient, such case does not reflect the real market dynamics as it corresponds to the situation when the average number of transactions on the time segment $(s, t]$ depends on length of this segment only. In our research, we would like to identify the difference between trade intensity throughout the day; therefore, we will assume that the measure $m$ has density, which is not constant:

$$
f(t):=(m([0, t]))^{\prime} .
$$

In other words, $m((s, t])=\int_{s}^{t} f(u) d u$ and, for any nonnegative integer $k$,

$$
\mathrm{P}\left(N_{t}-N_{s}=k\right)=\frac{e^{-\int_{s}^{t} f(u) d u}\left(\int_{s}^{t} f(u) d u\right)^{k}}{k !} .
$$

In what follows, the density $f$ will be referred to as the rate or intensity function.

\section{B. Nonparametric estimation of non-homogeneous Poisson process rate function}

In order to estimate $f$ from the real data, we use the kernel-based technique presented in [12] (for more detail on non-parametric estimation see also [13-14]). Let $N^{i}=\left\{N_{t}^{i}, t \in[0, T]\right\}, i=1, \ldots, M$, be $M$ independent trajectories of the Poisson process with intensity function $f$ and $K$ be the symmetric kernel with the compact support, i.e. the function which satisfies the following conditions:

- $\quad K$ is bounded and nonnegative;

- $\forall u \in(-\infty,+\infty), U: K(u)=0$, where $U$ is some compact set;

- $\forall u \in(-\infty,+\infty): K(u)=K(-u)$;

- $\quad \int_{\breve{y}} K(u) d u=1$

Then the estimator

$$
\mathcal{f}(t)=\frac{1}{M h} \sum_{i=1}^{M} \int_{0}^{T} K\left(\frac{t-u}{h}\right) N^{i}(d u)
$$

is the consistent estimator of the unknown intensity function $f$ (in sense of Theorem 6.4 from [12]) as $h \rightarrow 0$ and $M h \rightarrow \infty$.

\section{ANALYSIS OF DAILY TRANSACTION INTENSITY ON UKRAINIAN STOCK MARKET}

\section{A. Data}

To demonstrate the approach outlined in Section II, we analyze the transaction intensity on the Ukrainian stock market. We consider 6 risky assets traded on the Ukrainian Exchange [15] on the basis of which the UX index is calculated, namely shares of "Raiffeisen Bank Aval" (BAVL), "Centrenergo" (CEEN), "Donbasenergo" (DOEN), "MotorSich" (MSICH), "Turboatom" (TATM) and "Ukrnafta" (UNAF) (see Table I for more detail).

TABLE I. TRANSACTION INTENSITY ESTIMATION METADATA

\begin{tabular}{|c|c|c|c|}
\hline Name & Sample size & Starting date & Ending date \\
\hline BAVL & 11366 & 04.01 .2017 & 15.06 .2018 \\
\hline CEEN & 11843 & 04.01 .2017 & 15.06 .2018 \\
\hline DOEN & 5128 & 04.01 .2017 & 15.06 .2018 \\
\hline MSICH & 3343 & 05.01 .2017 & 20.04 .2018 \\
\hline TATM & 1256 & 04.01 .2017 & 15.06 .2018 \\
\hline UNAF & 12067 & 04.01 .2017 & 15.06 .2018 \\
\hline
\end{tabular}

Each observation contains information on the corresponding transaction including date and exact time. Therefore, it is possible to split the data into blocks corresponding to a separate day; the exact time of transaction execution can be considered as the point of jump of the Poisson process. 


\section{B. "The lunch-time effect" on the Ukrainian stock market}

First, we estimated the intensity rates using formula (2) for each asset from the list above, without any additional clustering. In such framework, $M$ is the number of trading days over the time indicated in Table I. Here and in what follows, we use the Epanechnikov kernel as kernel function, i.e. $K(u)=\frac{3}{4}\left(1-u^{2}\right) \mathbf{1}_{[-1,1]}(x)$. Fig. 2-3 contain plots of the intensity functions estimates for BAVL and UNAF assets respectively. Each graph is plotted using $R$ statistical software.

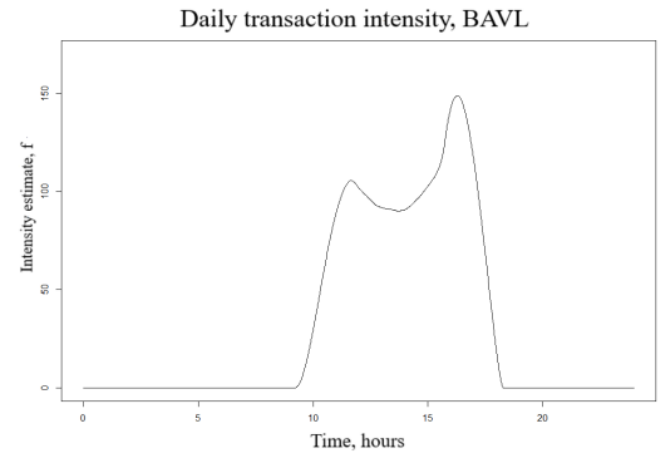

Fig. 2. Daily intensity function estimate for transaction occurrence, the BAVL asset.

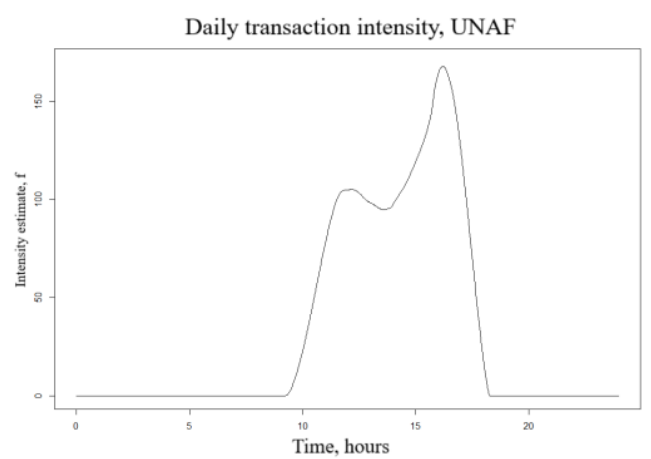

Fig. 3. Daily intensity function estimate for transaction occurrence, the UNAF asset.

As it can clearly be seen, the assumption concerning nonhomogeneity of the corresponding processes indeed reflects the real situation as the rate function differs from constant significantly. The most active time of the day on the market is its second part, from $3 \mathrm{pm}$ until closing. Another interesting phenomenon is a significant decrease in intensity in the middle of the day. It might be called "the lunch time effect" as the time gap when it occurs corresponds to a traditional lunchtime in Ukraine.

C. "Day-of-the-week" effect on the Ukrainian stock market

Similarly to temporal structure of the transaction occurrence intensity throughout the day, it is possible to consider its day-to-day changes as well. To do this, we cluster the daily Poisson trajectory observations into groups, each of which corresponds to a particular day of the week. Afterwards, we use (2) to estimate separately transaction occurrence intensities associated to all of the weekdays. The results of the estimation for BAVL and UNAF assets for Thursday and Friday are given on Fig. 4-7.

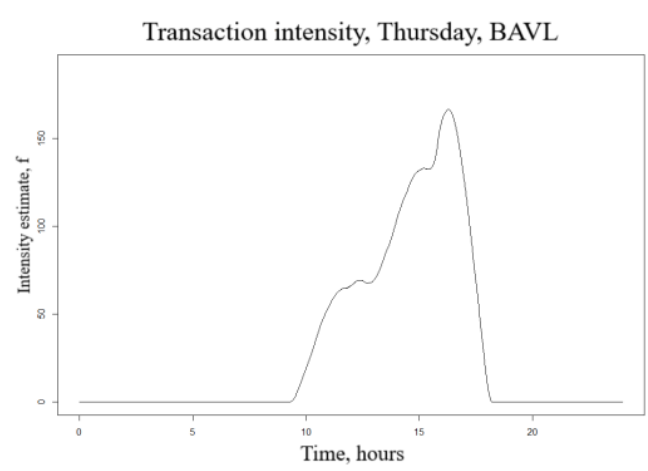

Fig. 4. Intensity function estimate for transaction occurrence, Thursday, the BAVL asset.

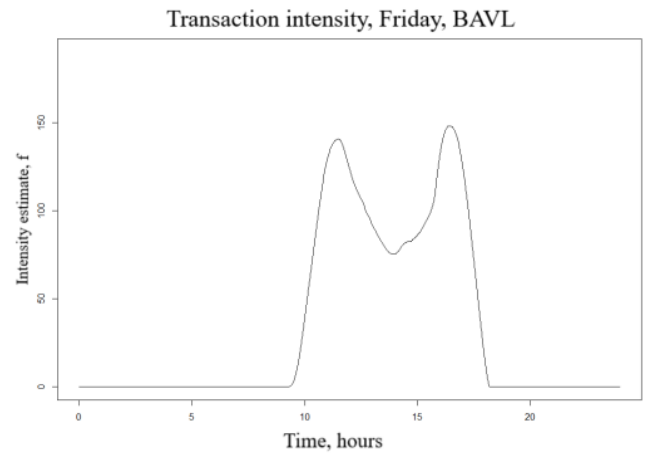

Fig. 5. Intensity function estimate for transaction occurrence, Friday, the BAVL asset.

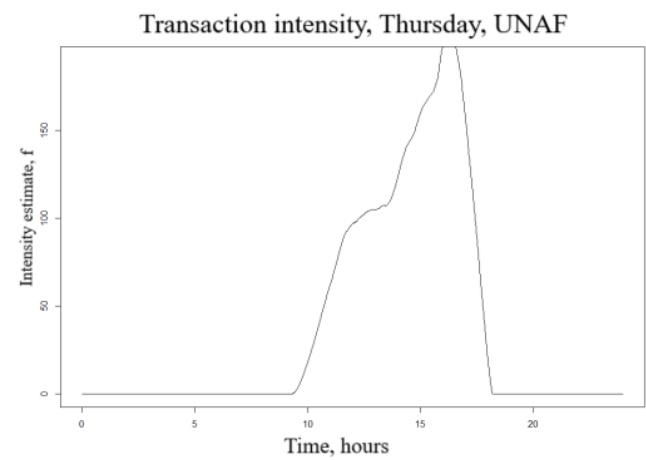

Fig. 6. Intensity function estimate for transaction occurrence, Thursday, the UNAF asset.

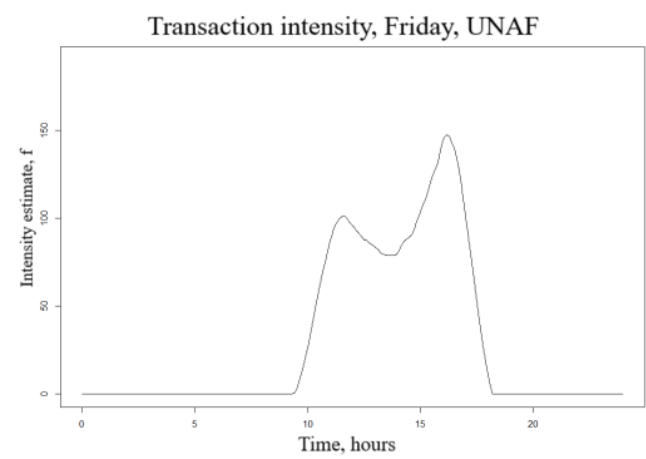

Fig. 7. Intensity function estimate for transaction occurrence, Friday, the UNAF asset. 
According to the plots, "day-of-the-week" effect is present on the Ukrainian stock market in transaction occurrence. For instance, the "lunch time" phenomenon described in the previous subsection can be clearly observed on Friday, while Thursdays are characterized by gradual increase in intensity during the day without any notable drops.

\section{INFLUENCE OF THE “DAY-OF-THE-WEEK” EFFECT ON TRANSACTION VOLUMES}

The Poisson process intensity function $f$ that was estimated in the previous section concerns events of transaction occurrence only, without reflecting the transaction volumes. As it has already been mentioned, the latter are modeled in (1) by the i.i.d. random variables $\xi_{i}$. In the context of previous section, the natural question arises whether distributions of random variables $\xi_{i}$ depend on the day of the week.

Fig. 8-9 depict box-and-whisker plots (with outliers removed) of volumes per transaction for the 3 of UX assets (TATM and MSICH), split into groups, which represent weekdays. As we can see, for different assets dependence may or may not occur, ranging from absence (MSICH, Fig. 8) to notable difference between weekdays (TATM, Fig. 9).

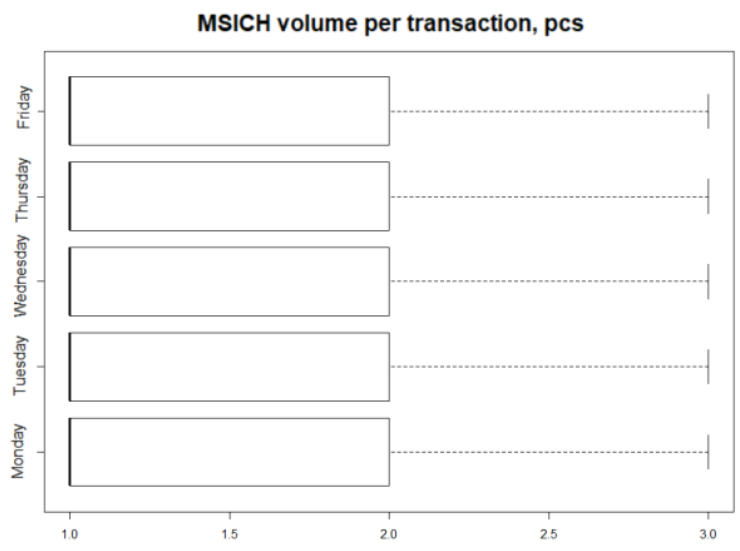

Fig. 8. MSICH volume per transaction, pcs

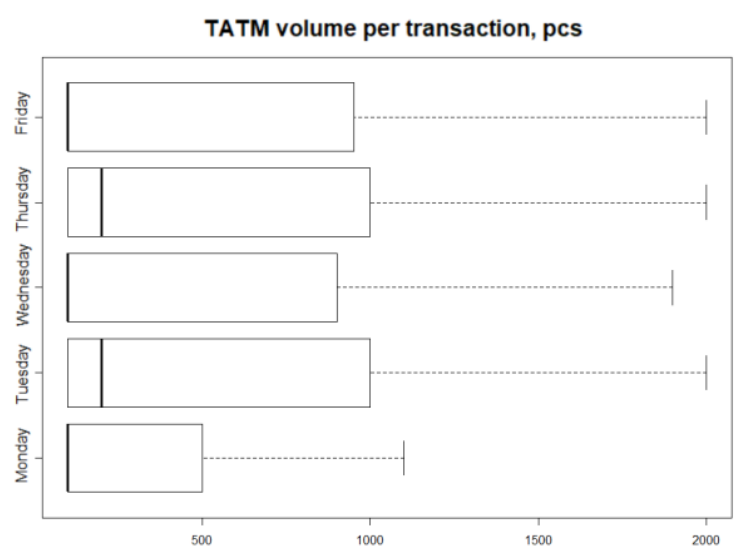

Fig. 9. TATM volume per transaction, pcs

\section{CONCLUSIONS}

In this research, we studied the transaction occurrence dynamics on the Ukrainian stock market using the technique based on intensity function estimation of the nonhomogeneous Poisson process. The chosen approach is new and has a significant advantage in comparison to classical linear time series methods: it allows to capture the trading intensity changes throughout the day as well as to compare the day-to-day trading rate.

As a result, several phenomena that happen on the Ukrainian stock exchange markets were studied, namely "the lunch time" and "day-of-the-week" effects. Such results reveal the influence of human factor on the market dynamics as the patterns found during the research strongly accord with sociological phenomena associated with the traditional schedule of residents of Eastern Europe.

Moreover, the results of the research clearly indicate that modeling of Ukrainian stock market dynamics requires dayto-day switching and cannot be characterized by a standard geometric Brownian motion process.

\section{REFERENCES}

[1] "Ukrainian Exchange - UX Index", Ukrainian Exchange, 2019. [Online]. Available: http://www.ux.ua/en/index/ux/. Accessed on: July 102019.

[2] "The National Securities and Stock Market Commission announces general indicators of the stock market index for 2018", Nssmc.gov.ua, 2019. [Online]. Available: https://www.nssmc.gov.ua/2019/02/14/nktspfr-ogoloshu-pokaznikifondovogo-rinku-za-2018-rk/?leng=eng\&testing=true. Accessed on: July 102019.

[3] K. French, "Stock returns and the weekend effect", Journal of Financial Economics, vol. 8, no. 1, pp. 55-69, 1980.

[4] H. Berument and H. Kiymaz, "The day of the week effect on stock market volatility", Journal of Economics and Finance, vol. 25, no. 2, pp. 181-193, 2001.

[5] H. Chen and V. Singal, "Role of Speculative Short Sales in Price Formation: The Case of the Weekend Effect", The Journal of Finance, vol. 58, no. 2, pp. 685-705, 2003.

[6] J. Zhang, Y. Lai and J. Lin, "The day-of-the-Week effects of stock markets in different countries", Finance Research Letters, vol. 20, pp. 47-62, 2017.

[7] G. Karanovic and B. Karanovic, "The Day-of-the-Week Effect: Evidence from Selected Balkan Markets", Scientific Annals of Economics and Business, vol. 65, no. 1, pp. 1-11, 2018.

[8] M. Iurchenko and A. Yurchenko-Tytarenko, "Statistical properties of the daily fluctuations of exchange rates of major world currencies (In Ukrainian)", in First national scientific-methodical conference in mathematical modeling in economics, Kyiv, 2016, pp. 397-399.

[9] Y. Mishura, and G. Shevchenko, Theory and Statistical Applications of Stochastic Processes. Hoboken: Wiley-ISTE, 2018.

[10] T. Rolski, H. Schmidli, V. Schmidt and J. Teugels, Stochastic Processes for Insurance and Finance. New York, NY: John Wiley \& Sons, 2009.

[11] T. Mikosch, Non-life insurance mathematics. Berlin: Springer, 2006.

[12] Y. Kutoyants, Statistical inference for spatial Poisson processes. New York: Springer, 1998

[13] W. Hardle, A. Werwatz, M. Müller and S. Sperlich, Nonparametric and Semiparametric Models. Berlin, Heidelberg: Springer Berlin Heidelberg, 2004.

[14] A. Tsybakov, Introduction to Nonparametric Estimation. New York: Springer Science+Business Media, LLC, 2009.

[15] "Ukrainian Exchange", Ukrainian Exchange, 2019. [Online]. Available: http://www.ux.ua/en/. July 102019 